\title{
The Mediterranean diet revisited: evidence of its effectiveness grows Francesco Sofi ${ }^{\mathrm{a}, \mathrm{b}, \mathrm{c}}$
}

aDepartment of Medical and Surgical Critical Care, Thrombosis Centre, University of Florence, ${ }^{\mathrm{b}}$ Regional Agency for Nutrition, Azienda Ospedaliero Universitaria Careggi and ${ }^{\mathrm{C}}$ Don Carlo Gnocchi Foundation, Onlus IRCCS, Impruneta, Florence, Italy

Correspondence to Francesco Sofi, MD, PhD, Department of Medical and Surgical Critical Care, Thrombosis Centre, University of Florence, Viale Morgagni 85, Florence 50134, Italy Tel: +39 055 7949420; fax: +39055 7949418; e-mail: francescosofi@gmail.com

Current Opinion in Cardiology 2009, 24:442-446

\section{Purpose of review}

The optimal dietary strategy for the prevention of chronic degenerative diseases remains a challenging and a highly relevant preventive health issue. In recent years, there have been ongoing investigations of the effectiveness of the Mediterranean diet in preventing the development of cardiovascular and other chronic diseases. Initial research suggested that the consumption of diets high in vegetable oil, fish, and complex grains and carbohydrates conferred a benefit in reducing the prevalence of cardiovascular disease. The purpose of this review is to examine the evidence in support of the effectiveness of the Mediterranean diet in reducing the prevalence of cardiovascular and chronic disease and their associated risk factors.

\section{Recent findings}

Recent studies intended to evaluate the Mediterranean diet in the primary or secondary prevention of cardiovascular and other chronic degenerative diseases have been focused on examining the impact of a whole dietary approach rather than on isolating single nutrients; it is recognized that analyses of single nutrients ignore the important and complex interactions between components of a diet and, more importantly, because people do not eat isolated nutrients. Therefore, dietary scores estimating the adherence to a Mediterranean diet within a population have been developed. Consumption of a Mediterranean diet has been found to be associated with a reduction of overall mortality and a reduced incidence of cardiovascular disease.

\section{Summary}

The Mediterranean diet has been consistently shown to be associated with favourable health outcomes and a better quality of life. The findings suggest that significant health gains can be expected in a general population whose diet reflects the nutritional principles reflected in a Mediterranean diet.

\section{Keywords}

cardiovascular diseases, health, Mediterranean diet, mortality

Curr Opin Cardiol 24:442-446

(C) 2009 Wolters Kluwer Health | Lippincott Williams \& Wilkins 0268-4705

\section{Introduction}

The relationship between nutrition and health has been studied intensively for more than half a century. Following the publication of the results of one of the landmark studies in this field, the Seven Countries' Study, the effect of nutrition on human health has been evaluated by many case-control, prospective cohort, and randomized clinical trials, all of which have provided evidence that diet may significantly affect the health state of a population [1,2]. An optimal diet, as a fundamental element of an array of 'lifestyle' issues, may significantly contribute to the maintenance of a healthy status. Conversely, poor-quality diets may favour the development of adverse health states including obesity, dyslipidaemia, hypertension (the combination of which may lead to the appearance of the 'metabolic syndrome'), and the emer- gence of chronic diseases such as diabetes, coronary artery disease, neoplastic, and neurodegenerative disorders. In recent years, major scientific and health associations have increasingly focused their chronic disease prevention strategies on the reduction of the global risk burden; in this scenario, a great importance is placed on matters relating to nutrition [3,4]. To date, Hippocrates' famous aphorism 'we are what we eat' seems more pertinent than ever. The evidence continues to accumulate that dietary quality and quantity are important determinants of health status. Cardiovascular disease remains the leading cause of death and disability among the industrialized countries, and there is understandable interest in encouraging dietary practices likely to minimize the likelihood of their development and progression. At present, only adoption of a Mediterranean-style dietary pattern has consistently shown an ability to offer 
Table 1 Food groups among the different Mediterranean countries

\begin{tabular}{|c|c|c|c|c|c|c|c|c|c|c|}
\hline & Bread & Pasta & Other cereals & Fruit & Vegetables & Fish & Legumes & Cheese & Wine & Olive oil \\
\hline Italy & $\sqrt{ }$ & $\sqrt{ }$ & + & ++ & ++ & ++ & + & + & ++ & $\sqrt{ }$ \\
\hline Greece & $\sqrt{y}$ & - & + & ++ & $\sqrt{ }$ & ++ & + & $\sqrt{ }$ & + & $\sqrt{ }$ \\
\hline France & $\sqrt{ }$ & - & + & + & ++ & + & + & $\sqrt{ }$ & $\sqrt{ }$ & ++ \\
\hline Spain & + & + & + & ++ & ++ & ++ & + & + & + & $\sqrt{ }$ \\
\hline North Africa & + & - & $\sqrt{ }$ & + & ++ & + & ++ & + & - & ++ \\
\hline East basin & + & - & ++ & + & ++ & + & + & + & - & ++ \\
\hline
\end{tabular}

- , not common; + , moderately common; ++ , highly common; $\sqrt{ }$, peculiar.

a degree of protection against the major chronic degenerative diseases.

\section{The Mediterranean diet: what is it?}

The definition of the Mediterranean diet dates back to the early 1960s, when Keys et al. [1] following journeys in southern Europe and the release of the Seven Countries' Study, described strikingly low rates of coronary heart disease in the Mediterranean countries when contrasted with other study populations [1]. Indeed, among the 14 populations examined within the seven countries, an approximately 10 -fold difference in rates of mortality for cardiovascular and neoplastic diseases was observed in favour of Mediterranean communities (Italy and Greece), in contrast to the other studied populations. Thereafter, the Mediterranean diet began to receive increasing attention from both scientific and lay communities; several studies have been conducted in order to investigate whether this dietary approach could be associated with a reduced risk of mortality and morbidity in a general population. The Mediterranean diet has been promoted as a model for healthy eating; it has been widely recognized to have favourable effects on lipid profile and to provide a significant source of antioxidants and vitamins. However, despite a worldwide promotion of this nutritional model, a progressive shift to westernised food habits has been occurring in southern European regions, and a loss of the original nutritional cultures is being observed [5]. In Italy, this trend has been strongly reflected by an increase in the prevalence of obesity over the past 10 years, especially among young people.

\section{The Mediterranean diet: further defined}

It is important to note that the Mediterranean-style diet is not a specific diet but rather a collection of eating habits traditionally followed by the populations of countries bordering the Mediterranean Sea. Keys originally described it as 'the dietary pattern of people living in Southern Italy in the 1960s'. The Mediterranean diet can be described as a dietary pattern characterized by high consumption of olive oil (as the prevalent visible fat), vegetables, legumes, whole grain products, fruits, and nuts. The intake of saturated animal fats is relatively low; a moderate fish consumption (varying with proximity to the sea) furnishes an adequate provision of polyunsaturated fats. Red wine, generally consumed during meals, is the principal source of alcohol. There is no single Mediterranean diet. The dietary practices of countries bordering the Mediterranean Sea vary considerably; even within the same country, significant differences in dietary pattern exist. Major differences in the traditional eating habits of citizens from countries bordering the Mediterranean Sea are noted in Table 1.

The Mediterranean diet should be not be considered as a dietary pattern but rather reflective of an integrated approach to living in which foods represent only one of the relevant cultural components, along with community beliefs, tradition, and the sense of pleasure associated with food preparation and intake. This complexity explains why a general definition of such a dietary pattern is difficult to develop, while revealing the challenges involved in assessing the elements and practices associated with adherence to a Mediterranean diet.

For decades, studies have attempted to evaluate the significance of isolated dietary components of the Mediterranean diet and their relationship with health outcomes. Literature has accumulated supporting the evidence that an increased consumption of food groups such as fruits and vegetables, fish, or whole-grain cereals is beneficial for health. Such analyses reflected the concept that, if a dietary pattern includes plenty of a specific food group (already shown to be associated with a reduced incidence of disease), the resulting diet will be beneficial to health. Such a reductionist approach presents several conceptual and methodological limitations not only because the components of any diet participate in an array of synergistic and antagonist interactions, but also, most importantly, because people eat a complex of nutrients and do not generally focus on isolated foods.

Moreover, the failure of several recent clinical trials of single nutrient supplementation suggests that it is the global nutrition pattern, rather than specific nutrients, that might have beneficial effects on the prevention of disease $[6,7]$. 
Thus, research efforts in this field have most recently reflected a switch to the evaluation of a score of adherence to the Mediterranean dietary pattern, rather than to the identification and evaluation of single nutrients in any single set of dietary behaviours.

As a result, an increasing number of studies have been carried out assessing, within any diet, the number of foods (or food practices) considered to be important for health. Such evaluations provide an overall measure of dietary quality, that is, a quality diet score.

\section{Evaluation of a Mediterranean diet}

Due to the aforementioned differences in dietary behaviours that exist among countries bordering the Mediterranean Sea and the lack of uniformity in diet content, it is easy to understand that there is no single direct method to quantify and measure the adherence to Mediterranean diet. A large number of indirect indices have been proposed. Two fundamental approaches have been commonly applied $\left[8^{\bullet}\right]$. One approach has been based on the composition of predefined quality indices using current nutrition recommendations from the major scientific and nutritional associations. This method is defined as the 'theoretically defined dietary adherence method'. The other is based on an 'empirical adherence to dietary patterns' and uses statistical approaches such as cluster analyses to describe and quantify adherence to a Mediterranean type diet.

The vast majority of the scores, to date, have been constructed based on the first approach, that is, using an evaluation of adherence to a theoretically, a-priori defined dietary pattern. Some specific indices have been widely used. Other modified approaches have also been introduced. The most commonly applied include the 'Mediterranean diet scale' [9], in its original and revised version, the 'MedDietScore' [10], and the 'Mediterranean Adequacy Index' [11]. Such dietary indexes have attempted to evaluate the complexity of a dietary pattern typically encountered in olive-growing areas. Using such indexes, individual diet quality can be ranked, and this approach has proven over the years to be a useful tool in the study of diet and its relationship to health.

Beginning in 1995, when the first study by Trichopoulou et al. [9] showed an association between an adherence index to Mediterranean diet and a clinical outcome, ongoing investigations have reported an association between greater adherence to a Mediterranean diet and a favourable health status. In 2003, findings from the Greek cohort of the European Prospective Investigation into Cancer and Nutrition (EPIC) study reported that a greater adherence to Mediterranean diet was associated with a lower all-cause mortality in an elderly population [12]. A two-point increase in a Mediterranean diet adherence score, as evaluated in a large cohort of more than 22000 elderly people, correlated with a $25 \%$ reduction in all-cause mortality. Two years later, the same authors confirmed their original findings noting a $18 \%$ lower overall mortality rate, when using a slightly modified diet score with specific reference to cardiovascular diseases [13]. Subsequently, an elevated adherence score to a Mediterranean diet has been linked to a reduction in inflammatory and coagulation markers, lipids, and blood pressure, the most important risk factors for cardiovascular diseases $\left[14-18,19^{\bullet \bullet}, 20\right]$.

In 2004, Chrysohoou et al. [14] demonstrated in a large population that patients who were in the highest tertile of a Mediterranean diet score had significantly lower levels of C-reactive protein, interleukin-6, white blood cell count, fibrinogen, and homocysteine. These findings have been confirmed by Fung et al. [15] who reported a significant inverse relationship between a higher Mediterranean diet score and C-reactive protein, interleukin6, E-selectin, and some markers of endothelial dysfunction. Very recently, the results of a multicentre, longitudinal study of 1003 myocardial infarction survivors from several European regions supported these findings, by showing a protective, inverse relationship between a Mediterranean diet score and the development of a proinflammatory state [16]. For each unit of increasing adherence to the Mediterranean diet, there was a reduction of $3.1 \%$ in the mean levels of $\mathrm{C}$-reactive protein and of $1.9 \%$ in the mean levels of interleukin-6.

Similarly, investigators assessing a 2-year randomized trial of a Mediterranean-style diet reported that it was effective in reducing C-reactive protein and interleukin-6 in individuals with metabolic syndrome [17]. Others have reported, following a 3-month randomized trial, that the traditional Mediterranean diet was more effective in reducing oxidized low-density lipoprotein (LDL)cholesterol levels than a low-fat diet [18]. These findings have been confirmed in a 2-year randomized trial conducted by Shai et al. [19** in a population of 322 moderately obese patients. In this study group, a Mediterranean diet resulted in significant weight loss and in a more favourable health status as reflected in improved risk parameters for cardiovascular disease: reduced LDLcholesterol levels and a lowering of the ratio of total to high-density lipoprotein (HDL) cholesterol. The Mediterranean diet was adjudged more effective than low-fat and low-carbohydrate diets in enhancing the cardiovascular risk profile.

Several investigations have demonstrated an association between a Mediterranean diet and reduced levels of classical cardiovascular disease risk factors such as hypertension, dyslipidaemia, and diabetes. In the Prevenciòn 
con Dieta Mediterranea (PREDIMED) study, the authors evaluated the effect of Mediterranean diet on the risk of cardiovascular disease in 772 men and women aged 55-80 years at high risk of cardiovascular disease; they reported decreased systolic $(-7.1 \mathrm{mmHg})$ and diastolic blood pressure $(-2.6 \mathrm{mmHg})$ after 3 months of follow-up in the Mediterranean diet group when compared with participants assigned to a low-fat diet [20].

These findings were confirmed in the results of The Seguimiento Universidad de Navarra study, another cohort prospective study that involved 9408 patients; a higher adherence to Mediterranean diet was associated with lower mean levels of systolic and diastolic blood pressure after 6 years of follow-up, though no association with a reduced risk of hypertension was observed [21].

Adherence to a Mediterranean diet has also been shown to be protective of other elements of the metabolic syndrome, such as diabetes. A prospective cohort study conducted in a population of 13380 Spanish university gradu-

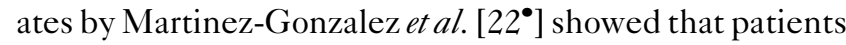
who adhered closely to a Mediterranean diet had a lower risk of developing diabetes over 4.4 years of follow-up; a two-point increase in the adherence score was associated with a $35 \%$-relative reduction in the risk of diabetes. In addition, a cross-sectional analysis of a subgroup of patients participating in the PREDIMED study with a high cardiovascular risk profile reported an inverse association between quartiles of adherence to Mediterranean diet and the prevalence of metabolic syndrome defined by the updated National Cholesterol Education Programme (NCEP)-III criteria [23]. Participants with the highest Mediterranean diet adherence had significantly lower odds ratio of having the metabolic syndrome in a population with a high risk of cardiovascular disease. A recent examination of the relationship between ingestion of a Mediterranean diet and cardiovascular risk factors among a large cohort of 3204 asymptomatic, high-risk patients demonstrated an inverse relationship between Mediterranean diet score and the presence of both single cardiovascular risk factors and their clustering [24 $4^{\circ}$. Adherence to a Mediterranean diet was related with an $18 \%$ reduction of being hypertensive, a $15 \%$ reduction of diabetes, a $16 \%$ reduction of being obese, and, importantly, a $33 \%$ reduced risk of the likelihood of possessing all three of these metabolic risk factors.

The first meta-analysis of all available studies evaluating a 'Mediterranean diet adherence score' and the incidence and mortality associated with major chronic degenerative disease has recently been completed $\left[25^{\circ \bullet}\right]$. The analysis of the results of 11 prospective, cohort studies involving more than 1.5 million participants showed that a greater adherence to the Mediterranean diet conferred significant protection against premature mortality from cardio- vascular and neoplastic diseases. The meta-analysis also demonstrated a positive protective effect of a Mediterranean diet in reducing the incidence of Parkinson's and Alzheimer's diseases. An increase of two points in the adherence score (easily achieved by increasing the consumption of some food groups characteristic of the Mediterranean dietary pattern) was shown to produce a $10 \%$ reduction in premature mortality or the incidence or both of major chronic degenerative diseases.

The recent publication of findings from the Nurses' Health Study, a cohort investigation comprising 74886 women aged 30-63 years followed for 20 years, confirmed the relationship between a Mediterranean diet and reduced rate of coronary heart disease [26 $\left.6^{\boldsymbol{\bullet}}\right]$. Women scoring in the highest quintile of the Mediterranean diet score were at lower risk for both coronary heart disease and stroke compared with those in the bottom quintile, with a demonstrated risk reduction of 29 and 13\%, respectively. Moreover, there was a significant, nearly $40 \%$ reduction in cardiovascular mortality experienced by women in the top quintile of the score.

\section{A public health perspective}

The evidence of the benefits of the Mediterranean diet has obvious clinical and public health relevance. The findings affirm the appropriateness of the recommendations and guidelines produced by many major scientific and clinical organizations which encourage the adoption of a Mediterranean-style diet as an approach to the primary and secondary prevention of major chronic diseases $[3,4]$.

Unfortunately, and ironically, despite worldwide promotion of the Mediterranean diet, there has been a gradual abandonment of this dietary pattern by the inhabitants of the Mediterranean basin (especially amongst the young) in recent years. Traditional food choices are changing because of the progressive globalization of food supply. Changes in eating behaviour and dietary choices are fundamental contributors to the rapid rise in overweight among some Mediterranean countries such as Greece and Italy. A recent analysis of the eating behaviours of 1305 children and adolescents from Greece demonstrated a very low adherence rate to Mediterranean diet [27]. The importance of initiating and maintaining positive dietary changes at the community level has long been recognized. The application of a Mediterranean diet adherence score, in many populations, may be helpful in further assessing and understanding the degree to which a community is modifying or improving its dietary habits.

\section{Conclusion}

In conclusion, an accumulating literature continues to indicate that the Mediterranean diet is one of the 
healthiest dietary models, not only in terms of a reduced incidence of some of the most important disease states, but also for enhancing overall life expectancy. It has recently been demonstrated that psychological well being and perceived health status are distinctly linked to diet and that consumption of a Mediterranean diet results in higher levels of perceived health [28]. For better mental and physical health, consider eating the Mediterranean way!!!

\section{References and recommended reading}

Papers of particular interest, published within the annual period of review, have been highlighted as:

- of special interest

-• of outstanding interest

Additional references related to this topic can also be found in the Current World Literature section in this issue (p. 504).

1 Keys A, Menotti A, Karvonen MJ, et al. The diet and 15-year death rate in the seven countries study. Am J Epidemiol 1986; 124:903-915.

2 Serra-Majem L, Roman B, Estruch R. Scientific evidence of interventions using the Mediterranean diet: a systematic review. Nutr Rev 2006; 64:S27S47.

3 Lichtenstein $\mathrm{AH}$, Appel $\mathrm{L}$, Brands $\mathrm{M}$, et al. American Heart Association Nutrition Committee. Diet and lifestyle recommendations revision 2006: a scientific statement from the American Heart Association Nutrition Committee. Circulation 2006; 114:82-96.

4 World Health Organization Study Group. Diet, nutrition, and the prevention of chronic diseases. Geneva, Switzerland: World Health Organization; Technical Report Series; 2003; 916.

5 Sofi F, Vecchio S, Giuliani G, et al. Dietary habits, lifestyle and cardiovascular risk factors in a clinically healthy Italian population: the 'Florence' diet is not Mediterranean. Eur J Clin Nutr 2005; 59:584-591.

6 Gaziano JM, Glynn RJ, Christen WG, et al. Vitamins E and C in the prevention of prostate and total cancer in men: the Physicians' Health Study II randomized controlled trial. JAMA 2009; 301:52-62.

7 Lee IM, Cook NR, Gaziano JM, et al. Vitamin E in the primary prevention of cardiovascular diseases and cancer: the Women's Health Study - a randomized controlled trial. JAMA 2005; 294:56-65.

8 Kourlaba G, Panagiotakos DB. Dietary quality indices and human health: a

- review. Maturitas 2009; 62:1-8.

A good review that reports all the differences among dietary quality indices in relation to health outcome.

9 Trichopoulou A, Kouris-Blazos A, Wahlqvist ML, et al. Diet and overall survival in elderly people. BMJ 1995; 311:1457-1460.

10 Panagiotakos DB, Pitsavos C, Arvaniti F, Stefanadis C. Adherence to the Mediterranean food pattern predicts the prevalence of hypertension, hypercholesterolemia, diabetes and obesity, among healthy adults: the accuracy of the MedDietScore. Prev Med 2007; 44:335-340.

11 Fidanza F, Alberti A, Lanti M, Menotti A. Mediterranean Adequacy Index: correlation with 25-year mortality from coronary heart disease in the Seven Countries Study. Nutr Metab Cardiovasc Dis 2004; 14:245248.

12 Trichopoulou A, Costacou T, Bamia C, Tricopoulou D. Adherence to a Mediterranean diet and survival in a Greek population. N Engl J Med 2003; 348:2599-2608.

13 Trichopoulou A, Bamia C, Tricopoulos D. Mediterranean diet and survival among patients with coronary heart disease in Greece. Arch Intern Med 2005; 165:929-935.
14 Chrysohoou C, Panagiotakos DB, Pitsavos C, et al. Adherence to the Mediterranean diet attenuates inflammation and coagulation process in healthy adults. The ATTICA study. J Am Coll Cardiol 2004; 44:152-158.

15 Fung TT, McCullough ML, Newby PK, et al. Diet-quality scores and plasma concentrations of markers of inflammation and endothelial dysfunction. Am J Clin Nutr 2005; 82:163-173.

16 Panagiotakos DB, Dimakopoulou K, Katsouyanni K, et al. Mediterranean diet and inflammatory response in myocardial infarction survivors. Int J Epidemiol 2009; 38:856-866. doi:10.1093/ijc/dyp142.

17 Esposito K, Marfella R, Ciotola M, et al. Effect of a Mediterranean-style diet on endothelial dysfunction and markers of vascular inflammation in the metabolic syndrome: a randomized trial. JAMA 2004; 292:1440-1446.

18 Fito M, Guzens M, Corella D, et al. Effect of a traditional Mediterranean diet in lipoprotein oxidation: a randomized controlled trial. Arch Intern Med 2007; 167:1195-1203.

19 Shai I, Schwarzfuchs D, Henkin Y, et al. Weight loss with a low-carbohydrate, -. Mediterranean, or low-fat diet. N Engl J Med 2008; 359:229-241.

A randomized controlled intervention trial in 322 moderately obese patients with Mediterranean diet compared with low-carbohydrate as well as low-fat diets. One of the longest randomized controlled intervention trials on Mediterranean diet and weight loss.

20 Estruch R, Martinez-Gonzalez MA, Corella D, et al. Effects of a Mediterranean diet on cardiovascular risk factors: a randomized trial. Ann Intern Med 2006; 145:1-11.

21 Nunez-Cordoba JM, Valencia-Serrano F, Toledo E, et al. The Mediterranean diet and incidence of hypertension. The Seguimiento Universidad de Navarra (SUN) study. Am J Epidemiol 2009; 169:339-346.

22 Martinez-Gonzalez MA, de la Fuente-Arrillaga C, Nunez-Cordoba JM, et al.

- Adherence to Mediterranean diet and risk of developing diabetes: prospective cohort study. BMJ 2008; 336:1348-1351.

A large cohort prospective study that assessed the relationship, over a 4-year follow-up period, between adherence to Mediterranean diet and the risk of developing diabetes.

23 Babio N, Bullo M, Basora J, et al. Adherence to the Mediterranean diet and risk of metabolic syndrome and its components. Nutr Metab Cardiovasc Dis 2009. [Epub ahead of print] doi:10.1016/j.numecd.2008.10.007.

24 Sanchez-Tainta A, Estruch R, Bullo M, et al. Adherence to a Mediterranean-

- type diet and reduced prevalence of clustered cardiovascular risk factors in a cohort of 3204 high-risk patients. Eur J Cardiovasc Prev Rehabil 2008; 15:589-593.

A cross-sectional assessment of the baseline characteristics of participants in the PREDIMED trial, investigating an association between adherence to Mediterranean diet and clustering of hypertension, diabetes, obesity, and hypercholesterolemia among high-risk patients.

25 Sofi F, Cesari F, Abbate R, et al. Adherence to Mediterranean diet and health -. status. A meta-analysis. BMJ 2008; 337:a1344.

This is the first meta-analysis that investigated the association between adherence score to Mediterranean diet and health status among cohort prospective studies. It analyses the relationship between adherence to Mediterranean diet and overall mortality from and incidence of cardiovascular diseases, neoplastic diseases, and chronic neurodegenerative diseases.

26 Fung TT, Rexrode KM, Mantzoros CS, et al. Mediterranean diet and incidence -. of and mortality from coronary heart disease and stroke in women. Circulation 2009; 119:1093-1100.

An updated analysis of the Nurses' Health Study, a large cohort prospective study with a follow-up of 20 years, with data of association between adherence score to Mediterranean diet and incidence of and mortality from coronary heart disease and stroke. This is the first study that investigated the association between Mediterranean diet and stroke in women.

27 Kontogianni MD, Vidra N, Farmaki AE, et al. Adherence rates to the Mediterranean diet are low in a representative sample of Greek children and adolescents. J Nutr 2008; 138:1951-1956.

28 Munoz MA, Fito M, Marrugat J, et al. Adherence to the Mediterranean diet is associated with better mental and physical health. Br J Nutr 2008; 15: $1-7$. 